\title{
BULLEIIN PREICEIIS DR PISCICLLTIRB
}

DECXIĖME ANNÉE

No 13

JULLET 1929

\section{CONSIDÉRATIONS \\ SUR LES RÈGIES BIOLOGIQUES DE L'ÉLEVAGE DES TRUITES ${ }^{(1)}$}

\author{
Par M. le Docteur Louis ROULE
}

Membre de lifiad mie d'A;reul'u:e, Prof sseur au Museum.

L'élevage des Truiles con:iitue l'une des deux branches les plus importantes de la pisciculture mo:lerne, l'autre branche étant représentée par l'élevage des Carpes en élangs. Il pârvient aujourd'hui, après une longrue période de tâtonnements et diessais, à un état de perfaction relative, où les salmoniculteurs commaisserit, de façon suffisante, la plupart des manipulations utiles qu'ils doivent accomplir. Mais cet état présente son danger. Sachant ce qu'ils doivent faire pour l'avoir vu pratiquer ailleurs, et n'ayant pas toujours procédé à l'étude rationnelle des recherches qui ont conduit à cette pratique, beaucoup d'entre eux omettent, parfois, de prendre los mesures convenables à la bonne conduite de leur industrie.

Ceci a pour résultat des mécomples assez fréquents. Ies établissements diffèrent entre eux par des qualités diverses, celle de l'eau, celle de l'installation, celle de la surveillance ef du contrôle, etc. lls ne sauraient se copier exactement. Ce qui convient a ux uns peut ne pas toujours convenir aux autres, et les opérations de la salmoniculture sont d'exécution délicate. Il est donc nécessaire aux éleveurs de pouvoir raisonner à l'avance leurs manipulations, d'en établir la marche et d'en prévoir les effets. Il en résulte, pour eux, l'obligation de connaitre les inclinations dominantes de la biologie normale des Truites, afin de se référer à clles pour ne point s'égarer, les erreurs se chiffrant, en ce cas, par des pertes souvent fort lourdes. Les considérations suivantes ont pour objet de préciser ces règles de biologie, auxquelles le salmoniculteur devra tenter de se conformer selon les moyens dont il peut disposer.

Ces règles principales portent :

Sur la nature des espèces élevées ;

Sur l'installation des bassins qui leur sont affectés :

Sur l'alimentation des ćlèves ;

Enfin sur la préparation de la fécondation artificielle.

( 1 Extrait d'un rapport présenté à la section de pisciculture du XIV Congrès inter: national d'Agriculture, tenu à Bucarest du 7 au ro juin 1929 . 
Elles sont exposées successivement et discutées ci-dessous selon cet ordre.

On s'est livré à de nombreux essais sur les espèces de Truites propres à l'élevage, dans le but de trouver les meilleures, plus résistantes que les autres, et de croissance plus rapide à égalité de soinz d'alimentation. On a étudié en ce sens la plupart des especes connues du genre Salmo et de ses voisins, y compris les hybrides que l'on a réussi à ubtenir et à élever. Actuellement, en mettant de côté les espèces et les varietés qui prévalent surtout par l'aspect ornemental, la majorité des éleveurs, du moins de ceux qui pratiquent la salmoniculture pour la consommation alimentaire directe et pour le repeuplement des cours d exu, se bornent à doux espèces : la Truite indigène (Salmo trulla L. ou Salmo fario L.) at la Truite arc-enciel (Salmo irideus Gibb. ou Trutle iridea Gibb.). Chacune delles ayant ses particularités propres d'exigrences biologiques, il en résulte qu'elles ne sont pas interchangeables et que le salmoniculteur doit s'adresser exclusirement à l'unc ou à l'autre scelon les résultats qu il désire obtenir.

La Truite indigène est d’un b́levagre délicat. Il hui faut une eau pure et très aérée, c'est-à-dire contenant en permanence une forte proportion d'orygène dissous, 6 à 7 centimètres cubes par litre au minimum, même pendant les fortes chaleurs du plein été. Flle est difficile à l'égard de son alimentation. Dans les conditions dc nature, elle se nourrit exclusivement de proies vivantes, et il lui faut une alimentation de cetle sorte, on s'en rapprochant le plus possible, jour que si croissance suive une courbe normale. Fourtant, elle est la seule qui convienne vraiment au repeuple. ment des cours deau, car elle reste à demeure dans ces derniers, et ne cherche point trop à les abandonner loregue !es conditions de milieu lui sont favorables.

Il n'en est pas de même, par contre, pour la Truite arc-en-ciel. Celle-ci, dans le cas d'immersion de ses alevins pour repeuplement, montre, sauf de rares exceptions sur lesquelles on ne doit pas se baser pour en conclure? à une règle générale, une propension évidente à rechercher les eaux profondes, à descendre les rivières oì on l'a immergée et, finalement, à disporâitre sans profit appréciable. Fun revanche, son élevage est plus avantageux que celui de l'autre espèce. Tout en ayant de grandes exigrences respiratoires, elle se contenic diune proportion plus faible d'oxygene dissous et peut tolérer, sans péricliter, le taux de ì à 6 centimètres cubes par litre. Cette facilité lui permettant de subsister dans des eaux relativement tièdes, il en résulte cue sa capacité d’assimilation cn est accrue, of que sia croissance est plus rapide.

La conclusion en est que, dans les élevages destinés à produire la Truite de consommation, il y a ávanlage à employer l'arc-en-ciel, et à s'en tenir à elle seule, sans chercher ailleurs. Par opposition, l'espèce indigène sera réservée aux établissements fondés pour obtenir des alevins de repeuplement. Les associations des cieux, parfois conseillées sous prétexte qu'elles 
s aident mutuellement dans leur entretien, donnent rarement les résultats que l'on en espérait.

Les Truites, dans la nature, fréquentent toujours des eaux vives, qu'elles soient courantes ou lacustres : c'est, dans leur biologie, leur habitat nor mal. L'expression " eaux vises ", souvent employée à cet égard, signifie simplement que ces eaux contiennent, sans discontinuité, la haute proportion d'oxygène dissous qui est indispensable à ces Poissons, comme à tous les Salmonidés du reste. I.curs exignences respiratoires priment toutes !es autres. Il est donc nécessaire au salmoniculteur d'y salisfaire et de tout mettre en ouvre pour at!eindre ce but.

On croit volontiers qu'il sutfit d'une rau quelconque, si elle est pure et abondante, pour avoir la base première d'un établissement d'élevage, Ces qualités, en effet, doivent être présentes ; mais à la condition rigoureuse de s'accompagner d'une autre, gui est la possession continue d'un taux d'oxygène dissous ne descendant pas au-dessous de 6 centimètres cubes par litre. Parfois un taux quelque pel inférieur peut être temporairement supporté, mais aux dépens de la santé des Poissons. Aussi le premier soin du salmoniculteur consistera-t-il à pratiquer l'analyse de l'eau dont il veut se servir, à en doser l'oxygène dissous, et à recommencer cette opéralion, à plusieurs reprises, en diverses saisons, pour connaître en ce sens toutes les variations possibles. Seule, cette constatation préliminaire lui permettra de savoir à quoi s'en tenir sur l'opportunité de l'emploi de cette eau dans son exploitation.

Malgré cette précaution préalable, et dans le cas où les conditions sont reconnues satisfaisantes, il peut arriver, accidentellement, que les eaux destinées à l'établissement, et réparties dans les bassins, se trouvent déficitaires quant à l'oxygène dissous. Ceci peut survenir, par exemple, pendant les périodes orageuses d'un été exceptionnellement chaud ou, encore, dans les établissements situés en montagne, et puisant leur eau dans une rivière, pendant la fonte des neiges. Aussi y a-t-il utilité à installer la canalisation de telle sorte que l'eau, avant de parvenir dans les bassins, tombe d'une certaine hauteur pour se brasser avec l'air ; on augmente ainsi, souvent de façon appréciable, la proportion de l'oxygène dissous.

Les anciens aménagements comportaient souvent la présence de filtres, destinés à arrêter les menus grains de vase en suspension, et à rendre l'eau plus pure. Ces appareils, autrelois, avaient leur utilité, car les incubateurs recevient de l'eau tombante, et la vase, s'il en existait, se déposait sur les œufs, dont elle entravait le développement. Cet inconvénient n'existe plus, on ne possède qu'une influence minime, dans les établissements modernes, dont les bacs d'incubation sont alimentés par de l'eau circulant de bas en haut, de manière à procéder à un nettoyage automatique et continu en entraînant les particules de vase. Les filtres, par conséquent, sont devenus inutiles. La cessation de leur emploi est à préconiser d'autant 
plus qu'ils étaient eux-mèmes, en raison de leur fonctionnement, de la stase de l'eau dans leur intéricur et des altérations subies par la vase déposée sur les matières filtrantes, des agents de diminution de l'oxygène dissous. Les fillres ont causé plus de mécomptes qu'ils n'ont procuré d'avantages.

Le nettoyage régulier des grands bassins et des étangs destinés à l'entretien des Truites de consommation et des reproducteurs ne devra pas être oublié. Des impuretés de toutes sortes, déchets de nourriture, excreta, cadavres, s'accumulent parfois dans des angles ou des cavités, et, par leur présence, contribuent à diminuer la proportion de l'oxygène respiratoire. Aussi est-il indispensable de maintenir partout la plus grande propreté. Les Poissons étant parqués en grand nombre dans un espace restreint, les conditions naturelles, où l'espacement des individus et l'abondance de l'eau procurent une aisance convenable de vie, se trouvent donc absentes, et il faut remédier à leur défaut par l'observation d'une hygiène piscicole teujours en éveil.

Les Truites, dans la nature, se nourrissent seulement de prores vivantes. Cunme il est impossible, dans l'élevage, où les indivirus se cumptent par inilljers et par dizaines de mille, de réunir les alimen!s de cetle sorte qui seraient nécessaires, on est obìgé de s'adruser a une nourrilure de rem placement. Les commodités économiques conduisent, dans ce but, la plupart des éleveurs à employer la pulpe de rate pour les alevins, et les déchets dir poissonnerie ou d'abattoirs, arec des farmes ile poisions comme succédanés, pour les pièces plus fortes exigeant une alimentation plus copieuse.

Cette nourriture, très différente de cellc qu'indique la biologie normale. comporte divers inconvénients, dont les principaux tiennent à l'usage alimentaire de chairs mortes, et au fait complémentaire que ces chairs, surtout pendant les chaleurs de l'été, et malgré les préservatifs d'antiseptiques ou de frigorification: ont subi un commencement de putréfaction. Il est donc utile de prévoir en ce sens les mécomptes possibles, afin de les attinuer, sinon les éviter.

Contre les menaces d'intoxication par des chairs ayant perdu leur frầcheur, la plupart des salmoniculteurs emploient la stérilisation à l'autoclave, ou plus simplement l'ébullition. ll est important, pour eux, de connaìtre que le pouvoir alimentaire des chairs ainsj traitées diminue dans des proportions considérables, et même de moitié. Les rations, par conséquent, devront être augmentées en conséquence.

On pourra pallier à l'inconvénient de l'ingestion de chairs mortes en mêlant à ces dernières, toutes lés fois où on le pourra, des chairs encore vivantes. Les salmoniculteurs qui, par leur situation ou leurs relations, peuvent se procurer des coquillages frais, et les utilisent de cette façon, en obtiennent de bons résultats. On a préconisé, récemment, l'emploi des huiles de poisson, riches en vitamines, dont on humecterait les pâtées avant de les distribuer. En ce qui concerne les Truites de consommation, 
dont les qualités de goût sont appréciées plus que toutes les autres, les chairs issues de proies fraîches méritent une meilleure considération.

Les éleveurs connaissent les avantages de la distribution régulière et du dosage des pâtées alimentaires. Malgré le rationnement judicieux, les Truites avalent souvent au delà de leurs besoins. La réplétion physiologique qui en résulte entraîne, si elle dure trop longtemps, des congestions viscérales, notamment du foie, dont la croissance ultérieure des individus et leur résistance aux agents pathogènes auront à subir l'effet. Aussi la pratique assez répandue, qui consiste à faire jeûner les Truites, une fois ou deux fois par semaine, pendant un jour entier, a-t-elle son avantage, car elle contribue à diminuer la valeur morbide de l'état de réplétion.

Il est un dernier point au sujet duquel l'observation des règles biologiques mérite d'être relenue : celui de la reproduction. A notre époque, où la pratique de la fécondation artificielle est d'usage courant, il est nécessaire de se rendre compte que l'on est parfois exposé à se servir de femelles dont la maturation sexuelle n'est pas encore achevéc. La mannuvre habituelle oblige pourtant les oxufs à ĉtre expulsés, ce qui n'aurait pas encore lieu dans la nature; et l'on féconde ainsi des oufs qui n'ont pas encore atteint leur pleine vigueur. Nautant que la maturation ayant lieu progressivement, et non pas simultanément, un certain nombre d'œufs trop jeunes peuvent se trouver mólangés aux autres. Ja fécondation aboutit pourtant à son résultat, et la plupart des ceufs deviennent embryonnés, arrivent même à la phase d'alevins vésiculés ; mais, plus tard, à l'époque de la résorption de la vésicule ainsi que dans celle des alevins de printemps, la mortalité, frappant surtout les produits issus d'œufs imparfaits, devient considérable. Ce sont lì des méromptes dont les salmoniculteurs ont parfois à souffrir durement.

Lá cause en est que la règle biologique n'a pas été observée. Dans la nature, les Truites femelles en instance de maturation sexuelle ne restent pas, d'ordinaire, dans les eaux ayant formé jusque-là leur habitat normal. Elles remontent les cours d'eau, pénètrent dans les petits ruisseaux latéraux, recherchent en somme un milieu plus richement pourvu d'oxygène dissous. Leurs échanges respiratoires passant alors par sa période de maximum, il leur faut, pour terminer la maturation et pouvoir pondre, cette sorte de coup de fouet final.

Il est donc nécessaire d'en faire autant, dans les élevages, pour les individus que l'on emploie au titre de reproducteurs. Au lieu de les laisser, et surtout les femelles, dans leur eau d'habitude, il convient de les parquer à l'avance dans un bassin dont les caux seraient les plus riches en oxygène dissous parmi celles dont l'établissement peut disposer. Grâce à cette condition, l'inconvénient signalé plus haut sera, sìon annihilé, du moins atténue. 